\title{
Long-term intake of soyabean phytosterols lowers serum TAG and NEFA concentrations, increases bile acid synthesis and protects against fatty liver development in dyslipidaemic hamsters
}

\author{
Sirle Laos ${ }^{1}$, Antoni Caimari ${ }^{1}$, Anna Crescenti ${ }^{1}$, Jamileh Lakkis ${ }^{2}$, Francesc Puiggròs ${ }^{1}$, Lluís Arola ${ }^{1,3}$ and \\ Josep Maria del Bas ${ }^{1 *}$ \\ ${ }^{1}$ Centre Tecnològic de Nutrició i Salut (CTNS), TECNIO, CEICS, Avinguda Universitat 1, 43204 Reus, Tarragona, Spain \\ ${ }^{2}$ Lipotec Company, Gavà, Barcelona, Spain \\ ${ }^{3}$ Nutrigenomics Research Group, Departament de Bioquímica i Biotecnologia, Universitat Rovira i Virgili,Tarragona, Spain \\ (Submitted 28 January 2014 - Final revision received 2 May 2014 - Accepted 8 May 2014 - First published online 16 June 2014)
}

\section{Abstract}

Various human trials and pre-clinical studies have suggested that dietary plant sterols possess hypotriacylglycerolaemic properties apart from their cholesterol-lowering properties. We hypothesised that phytosterols (PS) might attenuate triacylglycerolaemia by interfering with the deleterious effects of cholesterol overload in the liver. In the present study, twenty hamsters (Mesocricetus auratus) with dietinduced combined hyperlipidaemia were fed a high-fat diet (HFD, $n$ 10) or a HFD supplemented with soyabean PS ( $n$ 10) for $40 \mathrm{~d}$. In parallel, a healthy group was fed a standard diet $(n$ 10). PS normalised fasting plasma cholesterol concentrations completely after $20 \mathrm{~d}$ and were also able to normalise serum TAG and NEFA concentrations after $40 \mathrm{~d}$. HFD feeding caused microvesicular steatosis and impaired the expression of key genes related to fatty acid oxidation such as PPARA, carnitine palmitoyltransferase-I $\alpha(C P T 1 A)$ and phosphoenolpyruvate carboxykinase 1 (PCK1) in the liver. PS treatment completely protected against HFD-induced steatosis and resulted in a normalised hepatic gene expression profile. The protection of the hepatic function by PS was paralleled by increased faecal cholesterol excretion along with a 2 -fold increase in the biliary bile acid (BA):cholesterol ratio. The present study supports the conclusion that long-term consumption of PS can reduce serum TAG and NEFA concentrations and can protect against the development of fatty liver via different mechanisms, including the enhancement of BA synthesis. The results of the present study place these compounds as promising hepatoprotective agents against fatty liver and its derived pathologies.

Key words: Bile acids: Combined hyperlipidaemia: Fatty liver: Phytosterols: TAG: NEFA

The cholesterol-lowering properties of dietary phytosterols (PS) have been described in various animal models and in human trials. Currently, a daily intake of $2.5 \mathrm{~g}$ of plant PS or phytostanols is recommended to reduce the concentrations of LDL-cholesterol by up to $10 \%$, potentially decreasing the risk of $\mathrm{CVD}^{(1)}$. Despite the unsettled debate about the exact mechanisms underlying these effects, it is well accepted that sterols and stanols can act at different levels during cholesterol absorption, mainly by interfering with the absorption of cholesterol and displacing it from the mixed micelle ${ }^{(2)}$ or enterocyte metabolism, thus affecting the assembly of chylomicrons ${ }^{(3,4)}$ and the influx and efflux of cholesterol from enterocytes $^{(5-7)}$. However, another known mechanism for the elimination of cholesterol from the body is that via the bile acid (BA) synthesis pathway. In the liver, cholesterol can be oxidised by the cytochrome P450 enzyme cholesterol$7 \alpha$-hydroxylase (encoded by CYP7A1), the main controller of BA synthesis ${ }^{(8)}$. The hydroxylated cholesterol moiety is subsequently subjected to successive modifications resulting in different BA species, which get secreted into the gall bladder and eventually reach the duodenum. After recirculation into the liver through the so-called enterohepatic circulation, BA are eventually excreted from the body through the faeces $^{(9)}$. Little is known about the effects of PS on the BA synthesis pathway; it has been shown in hamsters that the administration of soyabean sterol esters increases the faecal excretion of neutral lipids and the output of BA as well as changes the BA composition of the gall bladder ${ }^{(10)}$. In another study carried out in rats, stigmasterol has been found to increase the output of cholesterol and BA in a dose-dependent

Abbreviations: BA, bile acids; $C Y P 7 A 1$, cholesterol-7 $\alpha$-hydroxylase; HFD, high-fat diet; PLS-DA, partial least-squares discriminant analysis; PS, phytosterols; SCD1, stearoyl-CoA desaturase-1; STD, standard diet.

*Corresponding author: Dr J. M. del Bas, fax +34977300 431, email josep.delbas@ctns.cat 
fashion $^{(11)}$. Interestingly, it has been reported that in humans the cholesterol-lowering response to plant sterol consumption is conditioned by the promoter variant $-204 \mathrm{~A}>\mathrm{C}$ of the $C Y P 7 A 1$ gene $^{(12)}$, indicating that in humans PS are more effective if a more active promoter controls $C Y P 7 A 1$. Nevertheless, more studies are required to elucidate whether BA synthesis represents a mechanism for quantitative cholesterol elimination from the body in response to dietary PS.

Different investigations have ascribed other beneficial effects to dietary plant sterols and stanols apart from their cholesterol-lowering effects. A recent meta-analysis based on twelve randomised controlled trials has concluded that PS therapy can reduce blood TAG concentrations in humans ${ }^{(13)}$. Other supporting studies carried out in pre-clinical models have shown that the administration of $\beta$-sitosterol and stigmasterol over a period of 6 weeks decreases plasma TAG concentrations in hamsters ${ }^{(4)}$. In C57/BL6 mice, the administration of a plant sterol blend has been found to reduce hepatic and plasma TAG concentrations, but, surprisingly, not plasma cholesterol concentrations ${ }^{(14)}$. Different mechanisms have been proposed to explain these effects; some authors have proposed that PS might block the absorption of fats ${ }^{(14)}$, while others have concluded that plant sterols can reduce the release of VLDL from the liver ${ }^{(15)}$ or affect the assembly of TAG in enterocytic chylomicrons ${ }^{(4)}$. Additionally, some studies have highlighted the protective effects of PS against insulin resistance $^{(16)}$, hyperglycaemia ${ }^{(17)}$ or obesity ${ }^{(18)}$, thus placing these compounds as potentially effective agents against insulin resistance or the metabolic syndrome ${ }^{(19)}$

Despite growing evidence supporting the beneficial effects of plant sterols and stanols on TAG metabolism along with their hypocholesterolaemic activity, little is known about the involvement of the liver in the hypotriacylglycerolaemic properties of these bioactive compounds. Due to the key role that the liver plays in the control of the synthesis and distribution of lipids, we hypothesised that PS might attenuate triacylglycerolaemia by affecting hepatic processes related to fatty acid metabolism. We chose the golden Syrian hamster as the experimental model due to the common features that this mammal shares with humans but not with rats or mice regarding hepatic cholesterol and lipoprotein metabolism ${ }^{(20,21)}$. Therefore, in the present study, we treated hamsters with combined dyslipidaemia with PS due to the hypotriacylglycerolaemic effects of PS in hypertriacylglycerolaemic subjects ${ }^{(22)}$. We found that PS normalised fasting blood TAG concentrations several days after attenuating the cholesterolaemia. The hypotriacylglycerolaemic effects of PS were accompanied by the apparent protection against fatty liver development and the impairment of hepatic lipogenesis and fatty acid oxidation programme as well. In addition, PS-treated hamsters had a highly increased total BA:cholesterol ratio in the gall bladder. Altogether, the present study demonstrated that PS might have other beneficial properties apart from their hypocholesterolaemic properties, such as prevention of alterations of the hepatic lipid metabolism and, consequently, protection against steatosis and the combined dyslipidaemia associated with the consumption of a high-cholesterol diet.

\section{Materials and methods}

\section{Animals}

The Animal Ethics Committee of the Universitat Rovira i Virgili (Tarragona, Spain) approved all the procedures. The animals used were 3-month-old male golden Syrian hamsters (Charles River Laboratories) weighing about $100 \mathrm{~g}$. The hamsters were housed individually in cages at $22^{\circ} \mathrm{C}$ under a $12 \mathrm{~h}$ light $-12 \mathrm{~h}$ dark cycle (lights on at 09.00 hours) and with free access to food and water. After an adaptation period of $4 \mathrm{~d}$, twenty hamsters were fed a high-fat diet (HFD) providing $21 \%$ of energy as fat and 0.1\% of cholesterol (D10051907; Research Diets, Inc.) for $15 \mathrm{~d}$ to induce combined dyslipidaemia and ten hamsters (standard diet (STD) group) were fed a normal fat diet (D10051906; Research Diets, Inc.) throughout the study. On day 15, blood samples were obtained by saphenous vein puncture and hamsters fed the HFD were further divided into two groups. Of these two groups, one ( $n$ 10, HFD) was fed the described HFD and the other ( $n$ 10, HFD+PS) was fed the same HFD supplemented with $1 \%$ of PS (D10051908; Research Diets, Inc.). The composition of diets is given in Table 1. According to the manufacturer (Lipofoods), the PS provided were in the dispersible form (Lipophytol $^{\circledR}$ ) and contained $23.3 \%$ campesterol, $0.4 \%$ campestanol, 28.1\% estigmasterol, $43 \% \quad \beta$-sitosterol and

Table 1. Composition of the diets used in the study

\begin{tabular}{|c|c|c|c|}
\hline & HFD & $\mathrm{HFD}+\mathrm{PS}$ & NFD \\
\hline Protein $(\mathrm{g} / \mathrm{kg})$ & 25 & 25 & 22 \\
\hline Carbohydrate $(\mathrm{g} / \mathrm{kg})$ & 60 & 60 & 65 \\
\hline Fat $(\mathrm{g} / \mathrm{kg})$ & 11 & 11 & 4 \\
\hline \multicolumn{4}{|l|}{ Energy } \\
\hline $\mathrm{kcal} / \mathrm{g}$ & 4 & 4 & 4 \\
\hline $\mathrm{kJ} / \mathrm{g}$ & 17 & 17 & 17 \\
\hline \multicolumn{4}{|l|}{ Ingredients $(\mathrm{g} / \mathrm{kg})$} \\
\hline Casein, 80 Mesh & 233 & 231 & 220 \\
\hline L-Cys & 3 & 3 & 3 \\
\hline Wheat starch & 298 & 295 & 386 \\
\hline Maltodextrin 10 & 106 & 105 & 100 \\
\hline Dextrose & 53 & 53 & 50 \\
\hline Sucrose & 106 & 105 & 100 \\
\hline Cellulose, BW200 & 53 & 42 & 50 \\
\hline Coconut oil, 76 & 5 & 5 & 8 \\
\hline Flaxseed oil & 4 & 4 & 5 \\
\hline Sunflower oil & 11 & 11 & 30 \\
\hline Lard & 75 & 75 & 0 \\
\hline Mineral mix S10022G & 37 & 37 & 35 \\
\hline Vitamin mix V10037 & 11 & 11 & 10 \\
\hline Choline bitartrate & 3 & 3 & 2 \\
\hline Cholesterol & 1 & 1 & 0 \\
\hline Lipophytol & 0 & 10 & 0 \\
\hline \multicolumn{4}{|l|}{ SFA } \\
\hline $\mathrm{g} / \mathrm{kg}$ & 30 & 30 & 11 \\
\hline$\%$ & 34 & 34 & 27 \\
\hline \multicolumn{4}{|l|}{ MUFA } \\
\hline $\mathrm{g} / \mathrm{kg}$ & 30 & 29 & 8 \\
\hline$\%$ & 33 & 33 & 20 \\
\hline \multicolumn{4}{|l|}{ PUFA } \\
\hline $\mathrm{g} / \mathrm{kg}$ & 30 & 30 & 22 \\
\hline$\%$ & 33 & 33 & 53 \\
\hline
\end{tabular}

HFD, high-fat diet; HFD + PS, high-fat diet with phytosterols; NFD, normal fat diet. 
$1 \%$ estigmastanol. According to the Reagan-Shaw formula ${ }^{(23)}$, the PS dose used in the present study is equivalent to a daily human intake value of $3.8 \mathrm{~g}$ and far below the doses tested for adverse effects ${ }^{(24)}$. Nevertheless, it has been shown that PS at high doses can decrease the bioavailability of $\beta$-carotene and $\alpha$-tocopherol, despite the relevance of the consequences being still not known ${ }^{(25)}$. Body weight and food intake were recorded every $4 \mathrm{~d}$, and additional blood sampling was done on day 36 of the experiment. Faeces were collected $48 \mathrm{~h}$ before killing the animals. On day 57 , hamsters were fasted for $6 \mathrm{~h}$ and anaesthetised with sodium pentobarbital and blood was collected by cardiac puncture. Plasma was obtained by centrifugation, and tissues were dissected, weighed, snapfrozen in liquid $\mathrm{N}_{2}$ and stored at $-80^{\circ} \mathrm{C}$ until further analyses. Before freezing, a portion of the liver was sectioned and fixed in $4 \%$ paraformaldehyde for $24 \mathrm{~h}$ and stored at $4^{\circ} \mathrm{C}$ in phosphate buffer for histological examination. Bile was obtained by gall bladder puncture, snap-frozen in liquid $\mathrm{N}_{2}$ and stored at $-80^{\circ} \mathrm{C}$.

\section{Gene expression analysis}

Total RNA from the liver and retroperitoneal white adipose tissue was extracted as described previously ${ }^{(26)}$. RNA quality was checked spectrophotometrically and by agarose gel electrophoresis. Primers for the different genes are listed in Table S1 (available online), and these were obtained from biomers.net. Real-time quantitative PCR using $\beta$-actin as the reference gene were carried out as described previously ${ }^{(26)}$.

\section{Plasma analysis}

Commercial enzymatic colorimetric kits were used for the determination of the concentrations of plasma glucose (intra-assay reliability, CV\%, 0.83-0.59; inter-assay reliability, CV\%, 1.58-1.50), TAG (intra-assay reliability, CV\%, 0.390.43 ; inter-assay reliability, $\mathrm{CV} \%, 3.62-3.60$ ) (QCA) and NEFA (intra-assay reliability, CV\%, 0.75-0.61; inter-assay reliability, CV\%, 0.75-4.91) (Wako). The concentrations of plasma total cholesterol, HDL-cholesterol and VLDL/LDLcholesterol were determined using a colorimetric kit according to the manufacturer's instructions (BioAssay Systems) (intraassay reliability, $\mathrm{CV} \%, 0.83-0.52$; inter-assay reliability, $\mathrm{CV} \%$, 0.75-4.2). The concentrations of plasma insulin were measured using a mouse/rat insulin ELISA kit (intra-assay reliability, CV\%, 0.9-8.4; inter-assay reliability, CV\%, 6.0-17.9) (Millipore Iberica S.A.). The Revised Quantitative Insulin Sensitivity Check Index (R-QUICKI) was computed as described previously ${ }^{(26)}$.

\section{Extraction and quantification of lipids}

Lipids were extracted from the liver $(80 \mathrm{mg}$ ) and dried faeces $(100 \mathrm{mg})$ of hamsters using the methods described previously $^{(26)}$. TAG and total phospholipids were quantified using colorimetric kits (QCA), and cholesterol was quantified by GC-MS/MS. Briefly, samples were lyophilised, ground and subjected to solid-liquid extraction using methanol- chloroform (2:1) as an extractant. Samples and cholesterol standards (Sigma Aldrich Química SA) were dried under $\mathrm{N}_{2}$ steam, derivatised with $N$-methyl- $N$-(trimethylsilyl)trifluoroacetamide and further diluted with hexane. Cholesterol analysis was carried out using a 7890A GC coupled to a 7890A QqQ/ MS (Agilent Technologies). Chromatographic column used was a HP-5MS 5\% phenyl 95\% polydimethylsiloxane (Agilent Technologies). He gas of $9.99995 \%$ purity was used as a carrier gas, at a constant flow of $1 \mathrm{ml} / \mathrm{min}$. Sample volume was $1 \mu \mathrm{l}$ and the total run time was $20 \mathrm{~min}$. Ionisation was done by electronic impact. Acquisition was done in selectedion monitoring (SIM) mode, using $329 \mathrm{~m} / z$ as a quantifier ion and 368 and $458 \mathrm{~m} / z$ as the qualifier ions. The reproducibility of the method was estimated with a relative standard deviation (\%) of 3.64. The retention of cholesterol was computed as the difference between ingested and excreted cholesterol amounts during $48 \mathrm{~h}$.

\section{Bile composition analysis}

Bile was used for the quantification of total BA by a method based on the $3 \alpha$-hydroxysteroid dehydrogenase activity (Spinreact) (intra-assay reliability, CV\%, 2.69-1.2; inter-assay reliability $\mathrm{CV} \%, 5 \cdot 17-1.33$ ) and cholesterol (intra-assay reliability, $\mathrm{CV} \%$, 0.71-1.08; inter-assay reliability, CV\%, 1.240.76) using colorimetric kits (Spinreact and QCA, respectively) according to the manufacturer's instructions.

\section{Liver histology}

Morphometric analysis of the liver was carried out as described previously ${ }^{(27)}$. Macrovesicular steatosis was evaluated according to the method of Brunt $e t a l .{ }^{(28)}$ by estimating the percentage of area covered by fat droplets using a score graded from 0 to 3 ( 0 , null; 1 , steatosis detected in up to $30 \%$ of the microscopic fields; 2 , steatosis detected in between 30 and $60 \%$ of the fields; and 3, steatosis detected in more than $60 \%$ of the fields).

\section{Statistical analysis}

Data are expressed as means with their standard errors. For endpoint measurements, differences among the three experimental groups were assessed using one-way ANOVA. The homogeneity of variance assumption was confirmed by Levene's test. When the homogeneity of variances was not confirmed, a Greenhouse-Geisser correction was applied for one-way ANOVA (where indicated). When the one-way ANOVA indicated significant differences among the groups, post boc contrasts were applied using the Bonferroni method. Results of the post hoc contrasts are shown in the figures and tables using lower-case letters. Student's $t$ test was used where indicated exclusively to compare the means of two experimental groups. In the case of parameters measured at different time points, a mixed-design ANOVA based on a univariate type III repeated-measures ANOVA was used, setting time as the within-subjects variable to assess the effects of the treatments over time and the three 
experimental groups (STD, HFD and HFD+PS) as the between-subjects variable. The homogeneity of variances was confirmed by Mauchly's test. Results of the repeatedmeasures ANOVA are reported in the figures with capital letters indicating significant differences between the measures (T), significant differences among the experimental groups (G) or a significant interaction between the two $(T \times G)$ where indicated. The level of statistical significance was set at $P<0.05$ (two-tailed) for all the tests. All the statistical tests were conducted using the software $\mathrm{R}$ version 3.1.0 for Windows (R Project for Statistical Computing).

\section{Multivariate clustering analysis}

Principal component analysis and partial least-squares discriminant analysis (PLS-DA) were carried out using the statistical software Multibase 2013 (Numerical Dynamics). The resulting scores for the three first components were plotted on a $3 \mathrm{D}$ representation using the software $\mathrm{R}$ version 3.1.0 for Windows (R Project for Statistical Computing).

\section{Results}

\section{Body and tissue weights}

No differences were observed in food intake among the experimental groups (data not shown). Body weight curves (Fig. 1(A)), compared using repeated-measures ANOVA, revealed significant changes with time $\left(F_{14,378}=27 \cdot 8\right.$, $P<0.001)$, but not among the experimental groups $\left(F_{2,27}=2 \cdot 34, P=0 \cdot 115\right)$. Nevertheless, no significant $\mathrm{T} \times \mathrm{G}$ interaction was observed $\left(F_{28,378}=4.05, P<0.001\right)$, as body weight changes were dependent on the diet throughout the study duration. At the end of the experiment, liver and different adipose tissues were dissected and weighed (Fig. 1(B)). The weight of the different adipose tissue depots in the HFD groups, either with or without PS, was slightly increased, but changes were not statistically significant as revealed by oneway ANOVA. In contrast, liver weights differed significantly among the experimental groups $\left(F_{2,27}=14.2, \quad P<0.001\right)$. The post boc contrasts indicated that the liver weights of the HFD group were significantly increased by $35 \%$ compared with those of the STD group $(P<0 \cdot 001)$ and the HFD+PS group $(P=0.009)$. No differences were observed between the HFD+PS and the STD groups $(P=0 \cdot 150)$. Liver enlargement observed in the HFD group was accompanied by a clear steatotic profile (Fig. 1(C)), but not in the other two groups. Accordingly, histological analysis (Fig. 1(D)) revealed grade 3 microvesicular steatosis with no apparent fibrosis in the livers of the HFD group, while the degree of steatosis in the HFD+ PS group was similar to that in the STD group. As expected, the hepatic lipid profile was different among the experimental groups (Fig. 1(E)). Significant differences ( $\left.F_{2,27}=25.64, \quad P<0.001\right)$ were observed in hepatic total cholesterol concentrations between the HFD and the other two groups ( $P<0 \cdot 001$ for both), though no significant differences could be observed between the STD and the HFD+PS groups $(P=1)$. Hepatic TAG concentrations were significantly different among the experimental groups $\left(F_{2,27}=7 \cdot 317\right.$, $P=0.003)$, with the HFD group having higher concentrations than the STD and the HFD+PS groups $(P=0.006$ and $P=0 \cdot 015$, respectively), but not between the HFD + PS and the STD groups $(P=1)$. The concentrations of phospholipids

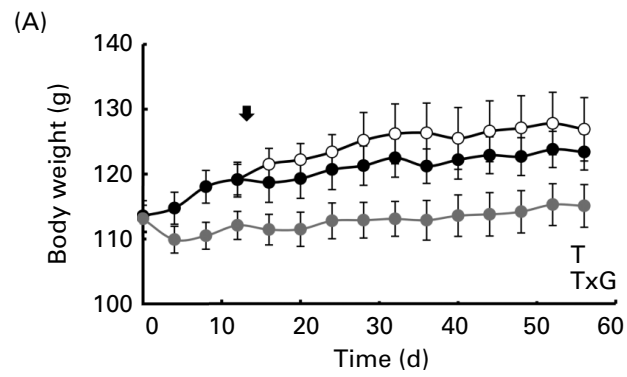

(B)

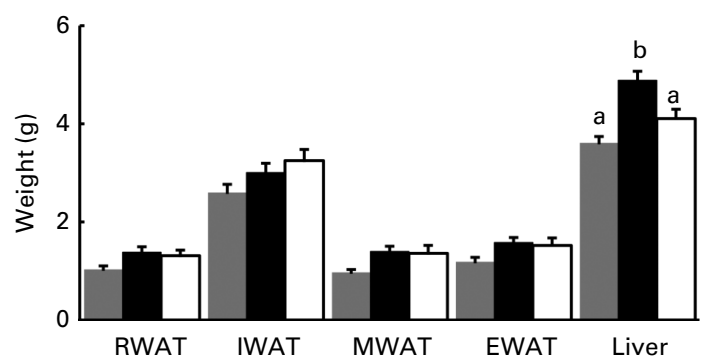

(C)

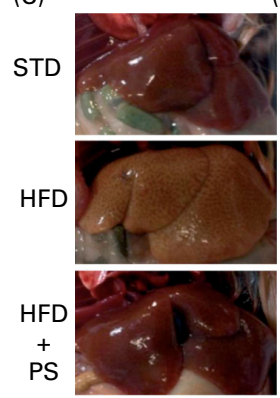

(D)

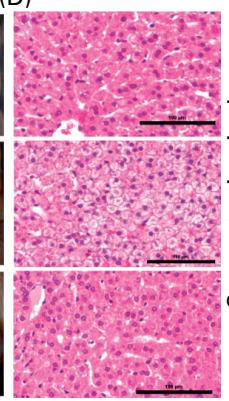

$(\mathrm{E})$

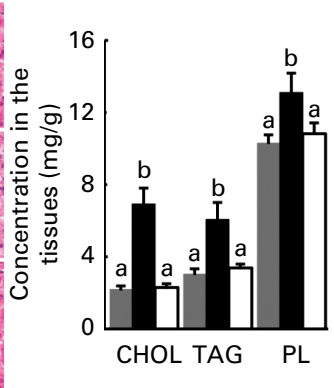

Fig. 1. (A) Evolution of body weight in hamsters fed a standard diet (STD, - - -) or a high-fat diet without phytosterols (HFD, - - ) or a HFD with phytosterols (HFD + PS, - - -). The arrow denotes the PS treatment starting point. Values are means $(n 10)$, with their standard errors represented by vertical bars. T denotes a significant effect of time $(P<0.001)$ and $T \times G$ denotes the significant interaction of time and group $(P<0.001)$ after a repeated-measures ANOVA comparison. Homogeneity of variances was confirmed by Mauchly's test. (B) Weights of the white adipose tissues (retroperitoneal white adipose tissue (RWAT), inguinal white adipose tissue (IWAT), mesenteric white adipose tissue (MWAT) and epididymal white adipose tissue (EWAT)) and livers of the STD $(\square)$, HFD $(\square)$ and HFD + PS $(\square)$ groups. Values are means ( $n 10)$, with their standard errors represented by vertical bars. ${ }^{a, b}$ Mean values with unlike letters were significantly different $(P<0.05$; Bonferroni post hoc test when one-way ANOVA revealed significant differences among the groups). Homogeneity of variances was confirmed by Levene's test. (C) Livers of the STD, HFD and HFD+PS groups (one animal representative of each group is shown). (D) Histology (haematoxylin and eosin staining, 20x, scale bar $100 \mu \mathrm{m}$ ) of representative liver sections of the STD, HFD and HFD + PS groups. (E) Hepatic concentrations of cholesterol (CHOL), TAG and phospholipids (PL). Values are means $(n 10)$, with their standard errors represented by vertical bars. ${ }^{a, b}$ Mean values with unlike letters were significantly different $(P<0.05$; Bonferroni post hoc test when one-way ANOVA revealed significant differences among the groups). Homogeneity of variances was confirmed by Levene's test. The level of statistical significance was set at $P<0.05$ (twotailed) for all the statistical tests. A colour version of this figure can be found online at http://www.journals.cambridge.org/bjn 
were significantly different among the experimental groups $\left(F_{2,27}=3.841, P=0.034\right)$, with the highest concentrations being detected in the HFD group than in the STD group $(P=0.033)$ and the HFD+PS group $(P=0.047)$, but not between the STD and the HFD+PS groups $(P=1)$.

\section{Plasma metabolites and cholesterol balance}

Plasma cholesterol and TAG concentrations were determined at three different time points (Fig. 2). On day 15, the HFD group exhibited a significant $(20 \%)$ increase in serum cholesterol concentrations $(P<0.001$; Student's $t$ test $)$ and a $35 \%$ increase in TAG concentrations $(P=0.003$; Student's $t$ test) compared with the STD group, confirming that the HFD animals had developed combined hyperlipidaemia. At that point, PS were introduced into the diets of the HFD+PS group for the remainder of the study duration. Repeatedmeasures ANOVA revealed serum total cholesterol (Fig. 2(A)) concentrations to be significantly different among the experimental groups $\left(F_{2,27}=20.7, P<0.001\right)$ and throughout the study duration $\left(F_{2,54}=4 \cdot 9, P=0 \cdot 01\right)$. A significant $\mathrm{T} \times \mathrm{G}$ interaction was observed $\left(F_{4,54}=10 \cdot 8, P<0 \cdot 001\right)$, indicating

(A)
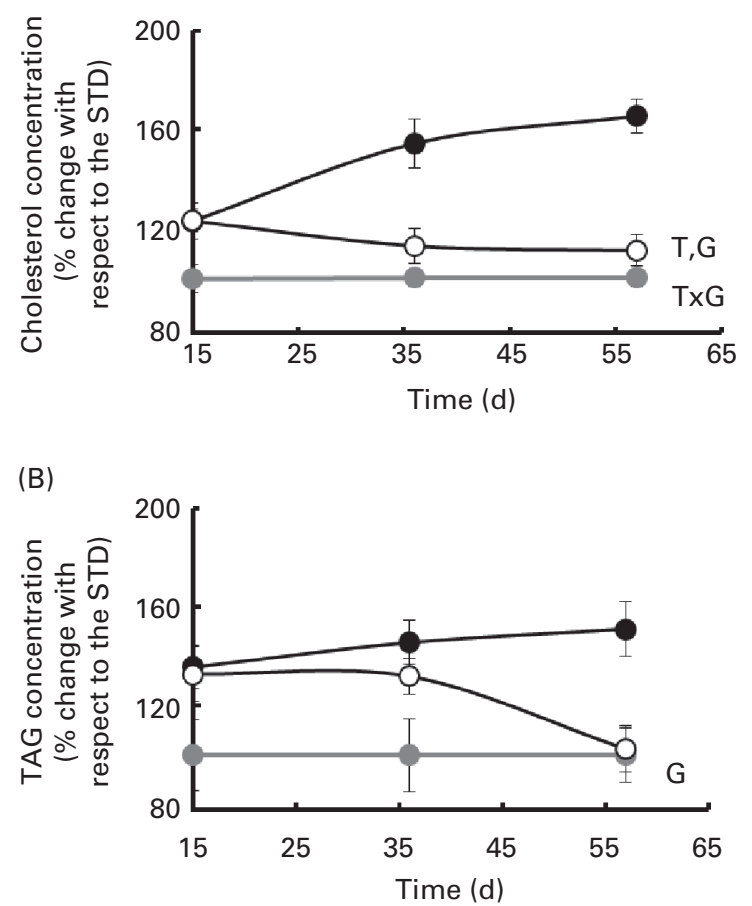

Fig. 2. Relative quantification of (A) serum cholesterol and (B) TAG concentrations in normolipidaemic hamsters (standard diet (STD), $\square$ ) and dyslipidaemic hamsters fed a high-fat diet without phytosterols (HFD, $\square$ ) or a HFD with phytosterols (HFD+PS, $\square$ ) on days 15, 36 and 57 of the experiment. Values are means $(n 10)$, with their standard errors represented by vertical bars. $\mathrm{T}$ denotes a significant effect of time $(P<0.001), \mathrm{G}$ denotes a significant effect among the experimental groups $(P<0.001)$, and $T \times G$ denotes the significant interaction of time and group $(P<0.001)$ after a repeatedmeasures ANOVA comparison. Homogeneity of variances was confirmed by Mauchly's test. The level of statistical significance was set at $P<0.05$ (two-tailed) for all the tests. that the change in cholesterol concentrations was dependent on the diet. Serum TAG concentrations (Fig. 2(B)) differed significantly among the experimental groups $\left(F_{2,27}=5 \cdot 0\right.$, $P=0 \cdot 01)$, but not throughout the study duration $\left(F_{2,54}=0 \cdot 2\right.$, $P=0.79)$, and no significant $\mathrm{T} \times \mathrm{G}$ interaction was observed.

Analysis of serum metabolites at the end of the experiment (Table 2) indicated significant changes in total cholesterol concentrations among the experimental groups $\left(F_{2,27}=36 \cdot 9\right.$, $P<0.001)$ due to increased concentrations being detected in the HFD group compared with those in the STD and the HFD + PS groups $(P<0.001$ for both), but not between the STD and the HFD+PS groups $(P=0 \cdot 31)$. The same pattern was observed for the concentrations of HDL-cholesterol $\left(F_{2,27}=4 \cdot 9, P=0.02\right)$ and VLDL + LDL-cholesterol $\left(F_{2,27}=19 \cdot 5\right.$, $P<0 \cdot 001)$. The post hoc contrasts confirmed that the differences were due to the HFD group, which had higher concentrations of all the fractions compared the STD and the HFD+PS groups ( $P<0.05$ for all the cases), while no differences were observed between the STD and the HFD+PS groups. Similarly, plasma TAG concentrations were found to be significantly altered $\left(F_{2,27}=7 \cdot 6, P=0.003\right)$. The post hoc contrasts indicated that the $56 \%$ increase in the HFD group was significantly higher than that in the STD and the HFD+PS groups $(P=0.005$ and $P=0.013$, respectively), but no significant differences were observed between the STD and the HFD+PS groups $(P=0 \cdot 98)$. No significant differences were observed in plasma glucose or insulin concentrations among these groups either. Nevertheless, plasma NEFA concentrations were significantly different among the experimental groups $\left(F_{2,27}=4.9, P=0.015\right)$, presumably due to the significantly lower concentrations in the HFD+PS group compared with those in the HFD group $(P=0 \cdot 012)$, while differences between the STD and the HFD groups can only be described as a trend $(P=0 \cdot 102)$. As a consequence, the insulin resistance index calculated as R-QUICKI indicated reduced insulin sensitivity in the HFD group with respect to the STD and the HFD+ PS groups, despite the fact that no statistical significance was revealed by one-way ANOVA.

Quantification of cholesterol in the diets and faeces (Table 3) revealed that cholesterol consumption patterns differed significantly among the experimental groups $\left(F_{2,27}=177\right.$, $P<0.001 ;$ Greenhouse-Geisser corrected). This difference was due to increased cholesterol consumption in the HFD and the HFD+PS groups compared with that in the STD group ( $P<0.001$ for both pairwise comparisons), while no differences were observed between the HFD and the HFD+PS groups $(P=0.92)$. Nevertheless, the significant change in cholesterol excretion $\left(F_{2,27}=63, P<0.001\right.$; Greenhouse-Geisser correction) was due to differences between the HFD+PS group and both the STD and the HFD groups $(P<0.001$ for both pairwise comparisons). These changes were reflected in differences in cholesterol retention $\left(F_{2,27}=64.7, P<0.001\right.$; GreenhouseGeisser correction), with the HFD+PS group displaying higher cholesterol retention than the STD group $(P=0.009)$, though lower than that in the HFD group $(P<0 \cdot 001)$. In turn, the HFD group also differed significantly from the STD group with respect to cholesterol retention $(P<0.001)$. 
Table 2. Plasma concentrations of metabolites in normolipidaemic hamsters fed a standard diet (STD) and dyslipidaemic hamsters fed a high-fat diet (HFD) and a HFD supplemented with phytosterols (HFD + PS)*

(Mean values with their standard errors, $n 10$ )

\begin{tabular}{|c|c|c|c|c|c|c|}
\hline & \multicolumn{2}{|c|}{ STD } & \multicolumn{2}{|c|}{ HFD } & \multicolumn{2}{|c|}{ HFD + PS } \\
\hline & Mean & SEM & Mean & SEM & Mean & SEM \\
\hline Glucose (mmol/l) & 14 & 0.6 & 15 & 0.5 & 14 & 0.6 \\
\hline Insulin (ng/ml) & $4 \cdot 8$ & 0.7 & $5 \cdot 7$ & 0.5 & 5.5 & 0.7 \\
\hline $\mathrm{NEFA}(\mathrm{mmol} / \mathrm{l})$ & $0.51^{a, b}$ & 0.04 & $0.59^{b}$ & 0.03 & $0.41^{\mathrm{a}}$ & 0.04 \\
\hline Free glycerol (mmol/l) & $0 \cdot 12$ & 0.01 & 0.14 & 0.02 & $0 \cdot 12$ & 0.02 \\
\hline TAG $(\mathrm{mmol} / \mathrm{l})$ & $1.3^{\mathrm{a}}$ & $0 \cdot 1$ & $2 \cdot 0^{\mathrm{b}}$ & 0.1 & $1.4^{\mathrm{a}}$ & 0.1 \\
\hline Total cholesterol $(\mathrm{mmol} / \mathrm{l})$ & $3 \cdot 9^{\mathrm{a}}$ & 0.3 & $6 \cdot 2^{\mathrm{b}}$ & 0.3 & $4 \cdot 2^{\mathrm{a}}$ & 0.3 \\
\hline HDL-cholesterol (mmol/l) & $3 \cdot 1^{\mathrm{a}}$ & 0.4 & $4 \cdot 2^{b}$ & 0.4 & $3 \cdot 2^{a}$ & 0.3 \\
\hline LDL + VLDL-cholesterol (mmol/l) & $0.9^{a}$ & 0.2 & $1.8^{\mathrm{b}}$ & 0.2 & $0.9^{a}$ & 0.1 \\
\hline R-QUICKI† & 0.243 & 0.005 & 0.231 & 0.002 & 0.245 & 0.004 \\
\hline
\end{tabular}

R-QUICKI, Revised Quantitative Insulin Sensitivity Check Index.

a,b Mean values within a row with unlike superscript letters were significantly different $(P<0.05$; one-way ANOVA using a Bonferroni post hoc contrast with a $\mathrm{Cl}$ of 0.95).

* Serum metabolite concentrations were determined at the end of the study after $6 \mathrm{~h}$ of fasting in blood samples collected by cardiac puncture.

†R-QUICKI was computed as indicated in the Materials and methods section.

\section{Expression of lipogenic genes in the liver and retroperitoneal white adipose tissue}

As has been reported, PS protected against hepatic lipid accumulation and liver enlargement induced by the HFD. Therefore, we analysed the expression of genes involved in fatty acid synthesis, esterification and oxidation. There were no changes in the expression of lipogenic genes acetyl-CoA carboxylase (ACC1) and diacylglycerol O-acyltransferase 2 (DGAT2) in the liver of all the experimental groups (Fig. 3(A)). In contrast, there were significant changes in the expression of fatty acid synthase $(F A S N)$ and stearoyl-CoA desaturase-1 (SCD1) $\left(F_{2,27}=5 \cdot 2, P=0.01\right.$ and $F_{2,27}=3 \cdot 9$, $P=0.03$; respectively). Despite applying the post hoc contrasts, no differences were observed in the expression of FASN between the HFD and the HFD+PS groups $(P=1)$; in the case of $S C D 1$, a clear tendency $(P=0.098)$ to recover the normal expression levels was observed in these groups. Clear differential effects were observed in the expression of $\beta$-oxidation-regulatory genes carnitine palmitoyltransferase-I $\beta$ (CPT1B) $\quad\left(F_{2,27}=6 \cdot 3, \quad P=0.006\right), \quad$ PPARA $\quad\left(F_{2,27}=12 \cdot 2\right.$, $P<0.001)$ and phosphoenolpyruvate carboxykinase
1 (PCK1) $\left(F_{2,27}=10 \cdot 8, P<0 \cdot 001\right)$. The post hoc contrasts indicated the HFD+PS group to differ significantly from the HFD group $(P<0.05)$ but not from the STD group with respect to the expression of these three genes. In contrast to our findings in the liver, no differences were observed in the expression of these genes in the retroperitoneal white adipose tissue between the HFD and the HFD+PS groups, despite a clear effect of the HFD either with or without PS being observed (Fig. 3(B)).

We also used a multivariate approach including the expression results of genes ACC1, FASN, DGAT2, SCD1, CPT1A, PPAR and PCK1 to assess the clustering trends of the animals. W chose a principal component analysis as an unsupervised (Fig. 4(A)) and a PLS-DA as a supervised (Fig. 4(B)) clustering analysis. The explained variance was $81.9 \%$ for the principal component analysis and $62.8 \%$ for the PLS-DA.

\section{Effects of phytosterols on the metabolism of bile acids}

The observed liver-specific changes prompted us to study an exclusive hepatic process tightly linked to lipid metabolism

Table 3. Cholesterol intake and excretion in normolipidaemic hamsters fed a standard diet (STD) and dyslipidaemic hamsters fed a high-fat diet (HFD) or a HFD supplemented with phytosterols (HFD $+\mathrm{PS})^{*} \dagger$ (Mean values with their standard errors, $n$ 10)

\begin{tabular}{|c|c|c|c|c|c|c|}
\hline & \multicolumn{2}{|c|}{ STD } & \multicolumn{2}{|c|}{ HFD } & \multicolumn{2}{|c|}{$H F D+P S$} \\
\hline & Mean & SEM & Mean & SEM & Mean & SEM \\
\hline Cholesterol intake (mg) & $0.70^{\mathrm{a}}$ & 0.04 & $7 \cdot 7^{\mathrm{b}}$ & 0.3 & $8 \cdot 2^{\mathrm{b}}$ & 0.4 \\
\hline Cholesterol excreted (mg) & $0 \cdot 13^{\mathrm{a}}$ & 0.02 & $0.3^{\mathrm{a}}$ & 0.03 & $6 \cdot 3^{b}$ & 0.5 \\
\hline Cholesterol retention (mg) & $0.57^{a}$ & 0.05 & $7.45^{\mathrm{b}}$ & 0.3 & $1.8^{\mathrm{C}}$ & 0.3 \\
\hline
\end{tabular}

${ }^{\mathrm{a}, \mathrm{b}, \mathrm{c}}$ Mean values within a row with unlike superscript letters were significantly different $(P<0.05$; one-way ANOVA using a Bonferroni post hoc contrast with a $\mathrm{Cl}$ of 0.95 ).

* Chow intake was monitored and faeces were collected for $48 \mathrm{~h}$. Cholesterol in the diets and faeces was quantified by GC.

†Data were analysed by a one-way ANOVA using a Greenhouse-Geisser correction when the homogeneity of variance assumption was discarded by Levene's test. 
(A)

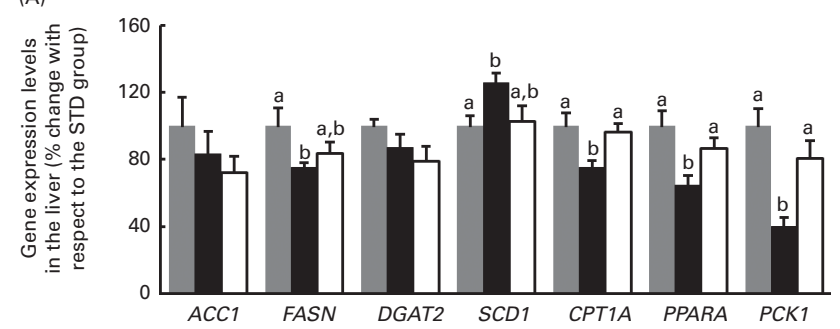

(B)

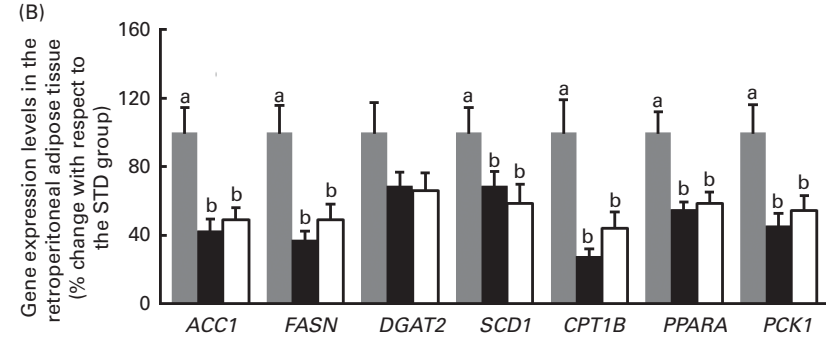

Fig. 3. Expression levels of genes related to fatty acid and glycerolipid metabolism in the (A) liver and in the (B) retroperitoneal adipose tissue of normolipidaemic hamsters (standard diet (STD), $\square$ ) and dyslipidaemic hamsters fed a high-fat diet without phytosterols (HFD, $\mathbf{c}$ ) or a HFD with phytosterols (HFD + PS, 口). Gene expression was quantified by real-time quantitative PCR using $\beta$-actin $(A C T B)$ as the endogenous control. Values are means $(n 10)$, with their standard errors represented by vertical bars. ${ }^{a, b}$ Mean values with unlike letters were significantly different $(P<0.05$; Bonferroni post hoc contrast when one-way ANOVA revealed significant differences among the groups). Homogeneity of variances was confirmed by Levene's test. The level of statistical significance was set at $P<0.05$ (two-tailed) for all the tests. The genes analysed were acetyl-CoA carboxylase $(A C C 1)$, fatty acid synthase $(F A S N)$, diacylglycerol $O$-acyltransferase 2 (DGAT2), stearoyl-CoA desaturase-1 (SCD1), carnitine palmitoyltransferase-l $\alpha(C P T 1 A)$, carnitine palmitoyltransferase-I $\beta$ (CPT1B), PPARA, and phosphoenolpyruvate carboxykinase 1 (PCK1).

and energy homeostasis such as BA synthesis. Analysis of the bile revealed that biliary cholesterol content (Fig. 5(A)) was significantly different among the experimental groups $\left(F_{2,27}=4 \cdot 8, P=0 \cdot 016\right)$, mainly due to differences between the HFD and the STD groups $(P=0.045)$, despite the fact that the HFD+PS group had lower, though non-significant, levels compared with the HFD group. Biliary total BA content (Fig. 5(B)) exhibited significant changes $\left(F_{2,27}=3 \cdot 4, P=0 \cdot 048\right)$ due to increased levels being detected in the HFD and the HFD+PS groups with respect to the STD group $(P=0.043$ and $P=0.042$, respectively). The BA:cholesterol ratio (Fig. 5(C)) was significantly affected $\left(F_{2,27}=6.4, P=0.005\right)$ mainly due to the 2 -fold increase in the ratio of the HFD+PS group compared with that of the HFD group $(P=0.006)$. We quantified the mRNA levels of hepatic $C Y P 7 A 1$ and found the expression of $C Y P 7 A 1$ to be 2 -fold higher in the HFD+PS group than in the HFD group (Fig. 5(D)), although these changes were not significant when the three experimental groups were compared using one-way ANOVA $\left(F_{2,27}=2 \cdot 45, P=0 \cdot 105\right)$. We also analysed the gene expression of farnesoid $\mathrm{X}$ receptor $(F X R)$, small heterodimer partner $(S H P)$ and hepatocyte nuclear factor- $4 \alpha$ (HNF4A). The expression of HNF4A was found to be significantly altered $\left(F_{2,27}=3.9, P=0.03\right)$, mainly in the STD and the HFD groups $(P=0.036)$, but no differences were observed between the HFD+PS and the HFD groups $(P=0 \cdot 149)$ or the STD group $(P=0 \cdot 987)$. In turn, the expression of FXR and SHP was not altered by PS.

\section{Discussion}

Different studies have shown that PS might have additional beneficial properties apart from their hypocholesterolaemic activity, mainly plasma TAG-lowering or insulin resistanceameliorating properties. In the present study, we investigated the potential beneficial effects of soyabean PS on hepatic and blood lipids of golden hamsters with diet-induced combined hyperlipidaemia.

Currently, it is well accepted that PS inhibit the intestinal absorption of dietary cholesterol ${ }^{(2)}$. The results of the present study are in good agreement with the different mechanisms proposed, as the inclusion of PS into the HFD resulted in a 20-fold increase in faecal cholesterol content. However, additionally, we found evidence pointing to a supplementary
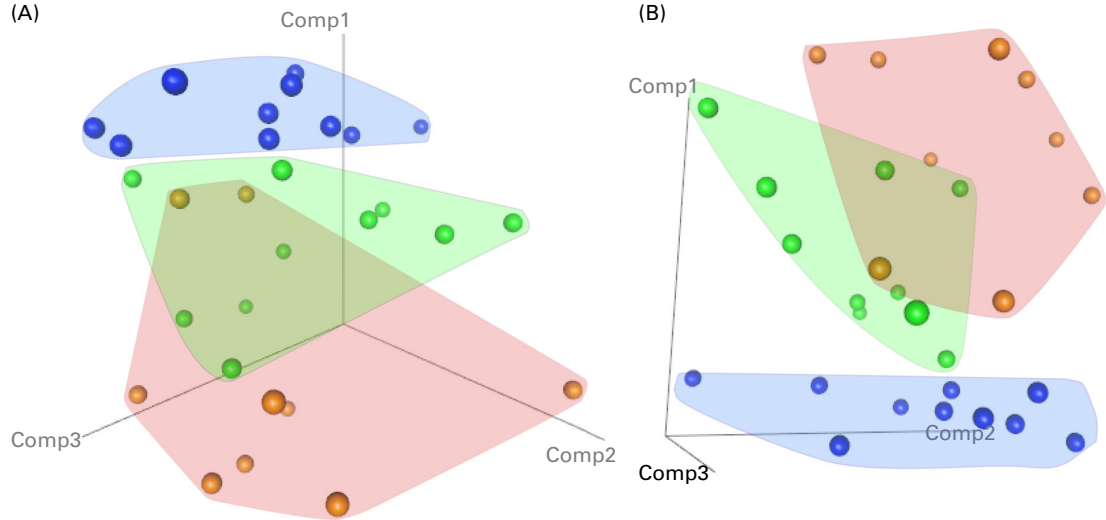

Fig. 4. Results of multivariate analysis of hepatic gene expression. Expression values of the genes acetyl-CoA carboxylase ( $A C C 1)$, fatty acid synthase ( $F A S N)$, diacylglycerol $O$-acyltransferase 2 (DGAT2), stearoyl-CoA desaturase-1 (SCD1), carnitine palmitoyltransferase-l $\alpha$ (CPT1A), PPAR and phosphoenolpyruvate carboxykinase 1 (PCK1) in the livers of normolipidaemic hamsters (STD, •) and dyslipidaemic hamsters fed a high-fat diet without phytosterols (HFD, ) or a HFD with phytosterols (HFD + PS, ) were used for an unsupervised clustering analysis by a principal components analysis (PCA) and for a supervised clustering analysis by a partial least-squares discriminant analysis (PLS-DA). The resulting scores for components (Comp) 1,2 and 3 of each animal are given for the (A) PCA and (B) PLS-DA. A colour version of this figure can be found online at http://www.journals.cambridge.org/bjn 
(A)

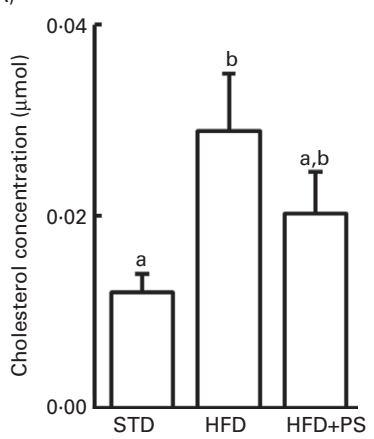

(B)

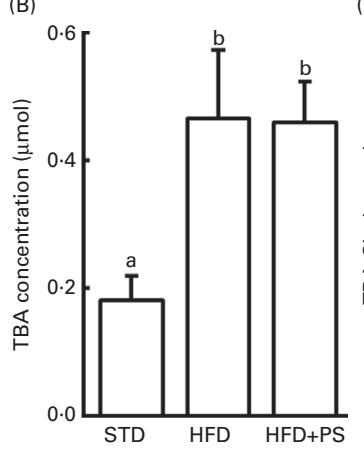

(C)

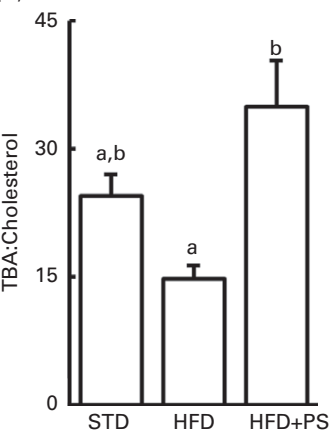

(D)

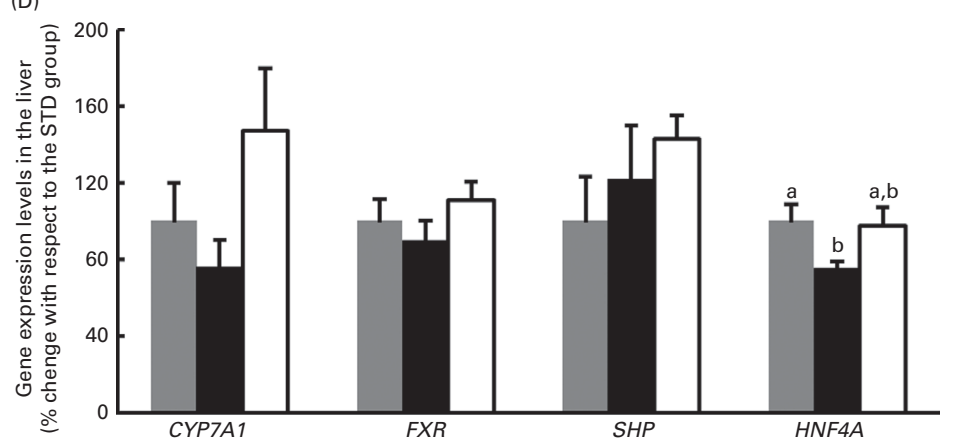

Fig. 5. Quantification of (A) cholesterol and (B) total bile acids (TBA) in the bile obtained from the gall bladder of normolipidaemic hamsters (STD) and dyslipidaemic hamsters fed a high-fat diet without phytosterols (HFD) or a HFD with phytosterols (HFD + PS). (C) TBA:total cholesterol ratio computed as described in the Materials and methods section. (D) Expression levels of genes related to bile acid metabolism in the liver of hamsters quantified by real-time quantitative PCR using $\beta$-actin $(A C T B))$ as the endogenous control. $\square$, STD; $\square$, HFD; $\square$, HFD + PS. Values are means ( $n$ 10), with their standard errors represented by vertical bars. ${ }^{\mathrm{a}, \mathrm{b}}$ Mean values with unlike letters were significantly different $(P<0.05$; Bonferroni post hoc test when one-way ANOVA revealed significant differences among the groups). Homogeneity of variances was confirmed by Levene's test. The level of statistical significance was set at $P<0.05$ (two-tailed) for all the tests. The genes analysed were cholesterol-7 $\alpha$-hydroxylase (CYP7A1), farnesoid X receptor $(F X R)$, small heterodimer partner $(S H P)$ and hepatocyte nuclear factor-4 $\alpha(H N F 4 A)$.

mechanism of action in the hamster. HFD feeding followed by PS supplementation in hamsters led to a remarkable increase in the biliary BA:cholesterol ratio, induced the expression of CYP7A1, which governs the synthesis of BA and controls its activity at the transcriptional level ${ }^{(8)}$, and normalised the expression of $H N F 4 A$, a key controller of BA metabolism ${ }^{(29-31)}$, indicating that PS enhanced BA synthesis. Additionally, it is worth considering that cholesterol retention in HFD+ PS animals was still higher than that in STD animals, while the hepatic cholesterol concentrations were similar in both groups. Altogether, these results indicate that the increased excretion of cholesterol via BA represents an additional mechanism of cholesterol reduction in the liver, thus explaining the striking protection against fatty liver development. In contrast to our findings, treatment of mice with $\beta$-sitosterol, which represents $38 \%$ of the PS used in the present experiment, has been found to decrease the faecal excretion of $\mathrm{BA}^{(32)}$. In Wistar-Kyoto and Wistar rats, stigmasterol, another PS present in the diets used in the present experiment, has been found to decrease hepatic cholesterol synthesis and, in parallel, reduce BA synthesis ${ }^{(33)}$. However, the results of the present study are in agreement with those of previous studies showing increased BA synthesis and altered BA composition in hamsters treated with soyabean sterol esters ${ }^{(10)}$. The possible explanation for the contrasting data can be the inherent differences in the animal models used. In hamsters and humans, the expression of $C Y P 7 A 1$ is not controlled by the concentrations of hepatic cholesterol as tightly as in rats and mice ${ }^{(34,35)}$. As expected, BA synthesis was not coupled to hepatic cholesterol concentrations in hamsters in the present study. As hamsters and humans share many common features related to cholesterol and BA metabolism $^{(21)}$, additional studies carried out in human subjects could shed some additional light on the effects of PS therapy on BA metabolism. Interestingly, it has been reported that the cholesterol-lowering response to PS intake in humans is conditioned by a more active variant $(-204 \mathrm{~A}>\mathrm{C})$ of the CYP7A1 gene promoter ${ }^{(12)}$. Therefore, the effectiveness of PS therapy in humans depends, at least in part, on the modulation of CYP7A1 gene expression. Nevertheless, it should be considered that the effects of PS on the excretion of cholesterol could take place through different pathways, such as dietary or hepatobiliary absorption to plasma as well as transintestinal cholesterol excretion $^{(2-7)}$. We currently do not know the contribution of the BA synthesis pathway to the observed effects of PS and whether these effects might be due to the direct or indirect actions of PS in the liver. Therefore, further research is required to determine the exact mechanisms, the quantitative contribution of each pathway and the relevance of the BA synthesis pathway to the effects of PS.

The HFD-fed animals had fatty liver as confirmed by the clear accumulation of lipids. This phenotype was accompanied by changes in the expression of genes that have previously been reported to be related to fatty liver in 
different models. It has previously been reported that mice lacking or with a low expression of hepatic PCK1 can develop severe fatty liver due to impaired control of energy homeostasis, involving the imbalance of cataplerotic and anaplerotic processes $^{(36)}$ and the subsequent impairment of $\beta$-oxidation. In the model used in the present study, HFD feeding resulted in a reduced expression of hepatic $\beta$-oxidation-regulatory genes, such as PPARA and CPT1A. Despite the debatable relevance of $\beta$-oxidation impairment in the development of fatty liver, the results of the present study indicate that this process might play an important role under the present experimental conditions. Together with these changes, a slight induction of SCD1 expression was observed, while the expression of other lipogenic genes remained unaltered. These results are in agreement with the results of previous studies showing that SCD1 is the lipogenic enzyme responding at the gene expression level in the fatty livers of golden hamsters ${ }^{(37)}$. A similar pattern was observed in the expression of $H N F 4 A$. It has been shown that this nuclear receptor is essential for several hepatic processes that were found to be altered in the model used in the present study, such as lipid synthesis, oxidation and secretion or BA synthesis ${ }^{(29,31)}$. These results can explain, at least partially, the excessive accumulation of TAG and phospholipids due to impaired fatty acid oxidation and increased fatty acid synthesis, together with excess dietary fat reaching the liver during the postprandial state. The addition of PS to the HFD normalised the hepatic lipid profile and the expression of the above-mentioned genes.

Differences in the expression of fatty acid metabolismrelated genes were statistically significant, but quantitatively mild when analysed individually. Therefore, we used two widely accepted multivariate clustering techniques, principal component analysis and PLS-DA, to assess whether the sum of these mild differences could be translated into clear contrasts. The outcome revealed that the HFD+PS-fed animals were partially similar to the STD-fed animals and significantly differed from the HFD-fed animals if all the analysed genes of fatty acid metabolism were used as clustering criteria. The clustering assay, therefore, reinforces and is consistent with the protection provided by the PS treatment against hepatic fatty acid metabolism. Explanation for these results could be found in the striking decrease in the hepatic cholesterol pool observed in the HFD+PS group. Previous studies have shown that a HFD and a high-fructose diet do not induce hepatic fat accumulation in hamsters, but that the addition of cholesterol to this background diet results in reduced PPAR $\alpha$ protein expression, increased lipogenesis and fatty liver development ${ }^{(37)}$. According to these data, a significant decrease in hepatic cholesterol pool is sufficient to prevent the development of fatty liver in hamsters. In fact, it has been suggested that excessive dietary cholesterol can trigger a cascade of events resulting in the impairment of hepatic fatty acid metabolism and, eventually, in fat accumulation in the liver of a broad range of mammalian species ${ }^{(38)}$.

Another relevant finding is the decrease in fasting plasma TAG and NEFA concentrations in the HFD+PS group with respect to the HFD group. This is in agreement with the results of previous studies in human subjects ${ }^{(13)}$ and hamsters ${ }^{(4)}$.
In the present experiment, the normalisation of fasting serum TAG concentrations by PS was a long-term effect, occurring between day 20 and day 40 of the treatment. In contrast, serum cholesterol concentrations decreased before day 20 of the treatment. These results indicate that the normalisation of plasma TAG concentrations by PS depends, at least in part, on the normalisation of hepatic lipogenesis and $\beta$-oxidation processes. In this scenario, the hepatic clearance of TAG in dyslipidaemic hamsters treated with PS might be slow, depending on fatty acid synthesis, increased fatty acid oxidation and the shuttling of TAG into VLDL for secretion. Consequently, this gradual removal of TAG might not be reflected in plasma VLDL concentrations until the hepatic concentrations of TAG are normalised. Nevertheless, other possibilities cannot be ruled out, as it has been shown that the intake of different stanols and sterols can modulate the mobilisation of hepatic fat. Thus, PS intake may enhance energy expenditure and hepatic $\beta$-oxidation in rats ${ }^{(18)}$. In humans, plant stanols are more efficient at reducing the release of TAG-rich VLDL from the liver in dyslipidaemic patients than in normolipidaemic subjects ${ }^{(15)}$ and, according to the authors, this observation fits with an enhancement of the hepatic $\beta$-oxidation programme. The results of the present study are in agreement with those of these studies, as PS caused a remarkable decrease in serum NEFA concentrations and a parallel increase in the expression of PPARA and CPT1A in the HFD-fed animals. Therefore, it is plausible to hypothesise that PS can enhance the oxidation of fatty acids in the liver, and perhaps in other tissues, contributing to a reduction of the overall pool of NEFA and TAG. Nevertheless, more research is still required to elucidate the exact mechanism leading to these effects. Increased concentrations of circulating NEFA have been proposed as a key factor for the development of insulin resistance ${ }^{(39)}$. Despite the suggested beneficial effects of PS consumption on insulin resistance via decreased circulating NEFA concentrations, more research is required to assess these effects.

Currently, combined hyperlipidaemia represents a risk factor for the development of CVD and is a hallmark of the metabolic syndrome, an altered state with a growing prevalence worldwide ${ }^{(40)}$. The prevalence of fatty liver or hepatic steatosis is also increasing, accompanied by other altered states such as obesity and/or insulin resistance ${ }^{(41)}$. The results of the present study, together with those of the other studies carried out in human subjects, indicate that long-term consumption of PS might provide remarkable protection to hepatic lipid metabolism against the deleterious effects of cholesterol and fatty acid overload, placing PS as effective agents against fatty liver and combined hyperlipidaemia development, including cholesterol, TAG and NEFA. At least two mechanisms could be responsible for these effects: first, increased faecal cholesterol excretion, which is in agreement with the classically accepted mechanism of action ascribed to PS and, second, the identified supplementary mechanism based on an increased efflux of cholesterol into the BA synthesis pathway. Even though we currently do not know the quantitative contribution of the BA synthesis pathway to the hypocholesterolaemic effects of PS, the results of the 
present study indicate that analysis of BA-related parameters in human trials including PS should be considered in future studies. In conclusion, the present study indicates that PS may be considered as promising agents in the treatment of lipid metabolism alterations beyond the reduction of blood cholesterol concentrations.

\section{Supplementary material}

To view supplementary material for this article, please visit http://dx.doi.org/10.1017/S0007114514001342

\section{Acknowledgements}

The authors cordially thank Mr David Moriñas for statistical support, Mr Ignasi Papell for management support and also Vanessa Grifoll and Silvia Pijuan, the laboratory technicians.

A. Caimari, J. L., L. A., F. P. and J. M. d. B. designed the research. S. L., A. Caimari, A. Crescenti and J. M. d. B. carried out the animal treatment, sample collection and analyses. J. M. d. B., A. Caimari and S. L. carried out the discussion of the results. J. L and J. M. d. B. wrote the paper.

J. L. is an employee of Lipotec Company. This does not alter the authors' adherence to all the British Journal of Nutrition policies on sharing data and materials.

\section{References}

1. Lauer MS \& Fontanarosa PB (2001) Updated guidelines for cholesterol management. JAMA 285, 2508-2509.

2. De Smet E, Mensink RP \& Plat J (2012) Effects of plant sterols and stanols on intestinal cholesterol metabolism: suggested mechanisms from past to present. Mol Nutr Food Res 56, 1058-1072.

3. Field FJ, Born E \& Mathur SN (1997) Effect of micellar $\beta$-sitosterol on cholesterol metabolism in $\mathrm{CaCo}-2$ cells. J Lipid Res 38, 348-360.

4. Liang YT, Wong WT, Guan L, et al. (2011) Effect of phytosterols and their oxidation products on lipoprotein profiles and vascular function in hamster fed a high cholesterol diet. Atherosclerosis 219, 124-133.

5. Plat J \& Mensink RP (2002) Increased intestinal ABCA1 expression contributes to the decrease in cholesterol absorption after plant stanol consumption. FASEB J 16, 1248-1253.

6. Zhao H, Houweling A, Vanstone C, et al. (2008) Genetic variation in ABCG5/G8 and NPC1L1 impact cholesterol response to plant sterols in hypercholesterolemic men. Lipids 43, 1155-1164.

7. Jia X, Ebine N, Demonty I, et al. (2007) Hypocholesterolaemic effects of plant sterol analogues are independent of ABCG5 and ABCG8 transporter expressions in hamsters. Br J Nutr 98, 550-555.

8. Davis RA, Miyake JH, Hui TY, et al. (2002) Regulation of cholesterol- $7 \alpha$-hydroxylase: BAREly missing a SHP. J Lipid Res 43, 533-543.

9. Alrefai WA \& Gill RK (2007) Bile acid transporters: structure, function, regulation and pathophysiological implications. Pharm Res 24, 1803-1823.

10. Carr TP, Cornelison RM, Illston BJ, et al. (2002) Plant sterols alter bile acid metabolism and reduce cholesterol absorption in hamsters fed a beef-based diet. Nutr Res 22, 745-754.
11. Andriamiarina R, Laraki L, Pelletier X, et al. (1989) Effects of stigmasterol-supplemented diets on fecal neutral sterols and bile acid excretion in rats. Ann Nutr Metab 33, 297-303.

12. De Castro-orós I, Pampín S, Mozas P, et al. (2011) Promoter variant $-204 \mathrm{~A}>\mathrm{C}$ of the cholesterol $7 \alpha$-hydroxylase gene? association with response to plant sterols in humans and increased transcriptional activity in transfected HepG2 cells. Clin Nutr 30, 239-246.

13. Demonty I, Ras RT, van der Knaap HCM, et al. (2013) The effect of plant sterols on serum triglyceride concentrations is dependent on baseline concentrations: a pooled analysis of 12 randomised controlled trials. Eur J Nutr 52, $153-160$.

14. Rideout TC, Harding SV \& Jones PJH (2010) Consumption of plant sterols reduces plasma and hepatic triglycerides and modulates the expression of lipid regulatory genes and de novo lipogenesis in C57BL/6J mice. Mol Nutr Food Res 54, Suppl. 1, S7-S13.

15. Plat J \& Mensink RP (2009) Plant stanol esters lower serum triacylglycerol concentrations via a reduced hepatic VLDL-1 production. Lipids 44, 1149-1153.

16. Tanaka M, Misawa E, Ito $\mathrm{Y}$, et al. (2006) Identification of five phytosterols from aloe vera gel as anti-diabetic compounds. Biol Pharm Bull 29, 1418-1422.

17. Alexander-Lindo RL, Morrison EYS \& Nair MG (2004) Hypoglycaemic effect of stigmast-4-en-3-one and its corresponding alcohol from the bark of Anacardium occidentale (cashew). Phytother Res 18, 403-407.

18. Ikeda I, Konno R, Shimizu T, et al. (2006) Campest-5-en-3-one, an oxidized derivative of campesterol, activates PPAR $\alpha$, promotes energy consumption and reduces visceral fat deposition in rats. Biochim Biophys Acta 1760, 800-807.

19. Nagao K \& Yanagita T (2008) Bioactive lipids in metabolic syndrome. Prog Lipid Res 47, 127-146.

20. Gao M, Zhang B, Liu J, et al. (2014) Generation of transgenic golden Syrian hamsters. Cell Res 24, 380-382.

21. Zhang Z, Wang H, Jiao R, et al. (2009) Choosing hamsters but not rats as a model for studying plasma cholesterollowering activity of functional foods. Mol Nutr Food Res 53, 921-930

22. Plat J, Brufau G, Dallinga-Thie GM, et al. (2009) A plant stanol yogurt drink alone or combined with a low-dose statin lowers serum triacylglycerol and non-HDL cholesterol in metabolic syndrome patients. J Nutr 139, 1143-1149.

23. Reagan-Shaw S, Nihal M \& Ahmad N (2008) Dose translation from animal to human studies revisited. FASEB $J \mathbf{2 2}$, $659-661$.

24. World Health Organization (2009) Evaluation of Certain Food Additives. Sixty-Ninth Report of the Joint FAO/WHO Expert Committee on Food Additives. WHO Technical Report Series no. 952. Geneva: World Health Organization.

25. Richelle M, Enslen M, Hager C, et al. (2004) Both free and esterified plant sterols reduce cholesterol absorption and the bioavailability of $\beta$-carotene and $\alpha$-tocopherol in normocholesterolemic humans. Am J Clin Nutr 80, 171-177.

26. Caimari A, del Bas JM, Crescenti A, et al. (2012) Low doses of grape seed procyanidins reduce adiposity and improve the plasma lipid profile in hamsters. Int $J$ Obes 37, 576-583.

27. García AP, Palou M, Sánchez J, et al. (2011) Moderate caloric restriction during gestation in rats alters adipose tissue sympathetic innervation and later adiposity in offspring. PLOS ONE 6, e17313.

28. Brunt EM, Janney CG, Di Bisceglie AM, et al. (1999) Nonalcoholic steatohepatitis: a proposal for grading and staging the histological lesions. Am J Gastroenterol 94, 2467-2474. 
29. Inoue $\mathrm{Y}$, Yu A-M, Yim SH, et al. (2006) Regulation of bile acid biosynthesis by hepatocyte nuclear factor $4 \alpha$. J Lipid Res 47, 215-227.

30. Kalaany NY \& Mangelsdorf DJ (2006) LXRS and FXR: the yin and yang of cholesterol and fat metabolism. Annu Rev Physiol 68, 159-191.

31. Hayhurst GP, Lee YH, Lambert G, et al. (2001) Hepatocyte nuclear factor $4 \alpha$ (nuclear receptor 2A1) is essential for maintenance of hepatic gene expression and lipid homeostasis. Mol Cell Biol 21, 1393-1403.

32. Uchida K, Takase H, Nomura Y, et al. (1984) Changes in biliary and fecal bile acids in mice after treatments with diosgenin and $\beta$-sitosterol. $J$ Lipid Res 25, 236-245.

33. Batta AK, Xu G, Honda A, et al. (2006) Stigmasterol reduces plasma cholesterol levels and inhibits hepatic synthesis and intestinal absorption in the rat. Metabolism 55, 292-299.

34. Horton JD, Cuthbert JA \& Spady DK (1995) Regulation of hepatic $7 \alpha$-hydroxylase expression and response to dietary cholesterol in the rat and hamster. J Biol Chem 270, 5381-5387.
35. Goodwin B, Watson MA, Kim H, et al. (2003) Differential regulation of rat and human CYP7A1 by the nuclear oxysterol receptor liver X receptor- $\alpha$. Mol Endocrinol 17, 386-394.

36. She P, Shiota M, Shelton KD, et al. (2000) Phosphoenolpyruvate carboxykinase is necessary for the integration of hepatic energy metabolism. Mol Cell Biol 20, 6508-6517.

37. Basciano H, Miller AE, Naples M, et al. (2009) Metabolic effects of dietary cholesterol in an animal model of insulin resistance and hepatic steatosis. Am J Physiol Endocrinol Metab 297, E462-E473.

38. Enjoji M, Yasutake K, Kohjima M, et al. (2012) Nutrition and nonalcoholic fatty liver disease: the significance of cholesterol. Int J Hepatol 2012, 925807.

39. Boden G (2011) Obesity, insulin resistance and free fatty acids. Curr Opin Endocrinol Diabetes Obes 18, 139-143.

40. Alberti KGMM, Zimmet P \& Shaw J (2005) The metabolic syndrome - a new worldwide definition. Lancet 366 , 1059-1062.

41. Farese RV Jr, Zechner R, Newgard CB, et al. (2012) The problem of establishing relationships between hepatic steatosis and hepatic insulin resistance. Cell Metab 15, 570-573. 